\title{
FAMÍLIA LAMIACEAE E A SUA INFLUÊNCIA NO DIABETES MELITUS: BREVE \\ REVISÃO
}

\section{LAMIACEAE FAMILY AND ITS INFLUENCE ON DIABETES MELLITUS: A BRIEF REVIEW}

\section{Amanda Migliorini Urban ${ }^{1}$, Juliane Nadal Dias Swiech², Marilis Dallarmi Miguel ${ }^{1}$}

1 - Universidade Federal do Paraná, Departamento de Farmácia.

2 - Centro de Ensino Superior dos Campos Gerais - Cescage,

Autor para correspondência: amandamurban@yahoo.com.br

\section{RESUMO:}

Estudos indicaram que as Lamiaceae possuem uma grande variedade de classes químicas que estão envolvidas na redução do nível de glicose no sangue. Grande número de espécies de plantas tem sido usado experimentalmente para tratar os sintomas do diabetes melito e a distância filogenética entre este grupo de plantas é forte indicação da natureza variada de seus constituintes. Tem sido constatado que muitas substâncias extraídas de plantas reduzem o nível de glicose no sangue. Sua grande diversidade de classes químicas indica que uma variedade de mecanismos de ação deve estar envolvida na redução do nível de glicose no sangue. Algumas destas substâncias podem ter potencial terapêutico enquanto outras podem produzir hipoglicemia como um efeito colateral devido à sua toxicidade, especialmente hepatotoxidade.

Palavras-chave: Produtos Naturais, Lamiaceae, Diabetes melitus

\section{ABSTRACT:}

Studies have indicated that Lamiaceae have a wide variety of chemical classes that are involved in lowering blood glucose levels. Large numbers of plant species have been used experimentally to treat the symptoms of diabetes mellitus and the phylogenetic distance between this group of plants is a strong indication of the varied nature of its constituents. It has been found that many substances extracted from plants reduce the level of glucose in the blood. Their wide diversity of chemical classes indicates that a variety of mechanisms of action must be involved in lowering blood glucose levels. Some of these substances may have therapeutic potential while others may produce hypoglycaemia as a side effect due to their toxicity, especially hepatotoxicity.

Keywords: Natural Products, Lamiaceae, Diabetes mellitus

\section{INTRODUÇÃO}

A maioria das espécies de plantas são utilizadas para fins médicos por milhares de anos. Estima-se que, cerca de 35000 a 70000 espécies tenham sido utilizadas em algum momento, por algumas culturas, com fins medicinais. A maior parte da população mundial representada pelos países em desenvolvimento, ainda dependem de ervas medicinais para suprir às suas necessidades de saúde. Nos serviços de saúde de primeira linha e básicos, 
ainda são prescritos frequentemente medicamentos à base de plantas, onde é o único serviço de saúde disponível, ainda assim, o interesse por essas ervas medicinais e a sua utilização vem crescendo rapidamente também nas áreas onde a medicina moderna é disponível (1998).

Com o uso crescente dessas ervas medicinais e seu maior interesse pela fitoterapia, criou-se a necessidade de uma melhor precisão no preparo e na avaliação, aumentando o estímulo a pesquisas com plantas, como seus usos e aplicações (1993), sendo de grande interesse para a indústria farmacêutica a sua elaboração do controle de qualidade e a padronização de medicamentos à base de plantas.

O Brasil é o país que possui a maior biodiversidade do planeta, que associada a uma rica diversidade étnica e cultural, detém um inestimável conhecimento tradicional associado ao uso de plantas com fins medicinais, possui também um potencial necessário para o desenvolvimento de pesquisas com resultados em tecnologias e terapêuticas oportunas. De acordo com a Organização Mundial da Saúde (OMS), o uso das plantas medicinais pela população de países mais pobres, remete cuidados especiais no seu uso através da distribuição de manuais para orientar estudos científicos que confirmem sua segurança e eficácia clínica (YUNES; CALIXTO, 2001).

Segundo a Lista de espécie da Flora do Brasil de 2020 em construção, já foram reconhecidas cerca de 46501 espécies da flora brasileira, sendo 4754 de Algas, 33100 de Angiospermas, 1567 de Briófitas, 5718 Fungos, 30 de Gimnospermas e 1332 de Samambaias e Licófitas (FLORA DO BRASIL, 2020), correspondendo entre 13-17\% da flora mundial (LEWINSOHN; PRADO, 2000).

A família das Lamiaceae possui espécies que são amplamente utilizadas, como o uso de óleos essenciais (Mentha, Lavandula, Marrubis, Nepeta, Ocimum, Origanum, Rosmarinus, Salvia, Satureja, Thymus, entre outras), tanto para utilização em cosméticos como condimentar, aromático e/ou medicinal e também são cultivadas como plantas ornamentais (SOUZA; LORENZI, 2012).

As espécies da família Lamiaceae acumulam substâncias com ampla variedade estrutural, apresentando esteróides, flavonoides, iridóides e terpenóides, atribuindo assim, várias atividades como: antitumoral, anti-HIV, anti-inflamatória, antioxidante, antibacteriana, antifúngica, anti-hipogligêmica, dentre outras (LEMES; FERRI, 2011). Esta breve revisão, tem o objetivo de compilar os benefícios que esta família possui principalmente quando falamos de doenças que são consideradas de saúde pública. 


\subsection{Família Lamiaceae}

A família Lamiaceae inclui cerca de 240 gêneros e 7200 espécies com distribuição cosmopolita (HARLEY; PASTORE, 2012). Ocorre principalmente em lugares abertos e em quase todos os tipos de habitats e em todas as altitudes, do Ártico ao Himalaia, do sudeste da Ásia ao Havaí; na Austrália, África, e de norte a sul no Novo Mundo, sendo o Mediterrâneo e a Ásia Central as regiões de maior concentração de espécies (HEYWOOD, 1979; SUDDE). No Brasil, ocorrem 34 gêneros nativos e cerca de 496 espécies (HARLEY, et al., 2010), muitas delas introduzidas como ornamentais ou para usos na culinária e até como madeira (HARLEY; FRANÇA, 2009).

Atualmente, a família está posicionada no clado Euasteridae I, grupo Lamiídeas, ordem Lamiales e apresenta sete subfamílias: Ajugoideae Kosteletzky, Lamioideae Harley, Nepetoideae Kosteletzky, Prostantheroideae Luerson, Scutellarioideae Caruel, Symphorematoideae Briquet e Viticoideae Briquet. A subfamília Nepetoideae é monofilética e apresenta estilete completamente ginobásico, suportado por pólens hexacolpados, endosperma ausente e seus óleos essenciais são constituídos por terpenoides muito voláteis e sequência de rbcL (JUDD et al., 2009). Esta subfamília é dividida em treze tribos: Nepeteae, Prunelleae, Glechoneae, Mentheae Rosmarineae, Lavanduleae, Hormineae, Monardeae, Salvieae, Meriandreae, Lepechinieae Elsholtzieae, Ocimeae e três subtribos: Hyptidinae, Plectranthinae e Ociminae. Compõe-se de 26 gêneros: Glecoma L., Dicerandra Benth, Hyptis Jacq., Rhaphiodon Schauer, Peltodon Pohl, Marsypianthes Mart. Ex Benth, Eriope Humb. \& Bonpl. ex Benth, Hyptidendron Harley, Eriopidion Harley, Hypenia (Mart. ex Benth.) Harley, Lycopus L., Melissa L., Mentha L., Monarda L., Nepeta L., Ocimum L., Origanum L., PlectranthusL'Hér., Prunella L., Pycnostachys Hook., Salvia L., Satureja L., Lavandula L., Thymus L. e Basilicum Moench (JUDD, et al., 2009).

A tribo Ocimeae é caracterizada por apresentar estames declinados e anteras sintecas; possui aproximadamente 35 gêneros e cerca de 1000 espécies, distribuída principalmente nas regiões tropicais e subtropicais, sendo seus principais centros de diversidade a América do Sul, a África Tropical, Madagascar e a China (PATON; RYDING, 1998).

A subtribo Hyptidinae é caracterizada por flores dispostas em cimeiras bracteoladas de diferentes tipos e os estames de currentes estão protegidos no lobo inferior da corola, sendo essa característica atribuída ao mecanismo de polinização explosiva (HARLEY, 1971). A subtribo possui, ainda, núculas com auréolas expandidas (PATON; RYDING, 
1998). Compõe-se de dezenove gêneros neotropicais: Asterohyptis Epling, Physominthe Harley \& J.F.B. Pastore, Hypenia (Mart. ex Benth.) Harley, Eriope Humb. \& Bonpl. ex Benth., Eriopidion Harley, Marsypianthes Mart. ex Benth., Rhaphiodon Schauer, Oocephalus (Benth.) Harley \& J.F.B.Pastore, Medusantha Harley \& J.F.B. Pastore ex Benth, Hyptis Jacq., Cyanocephalus (Pohl ex Benth.) Harley \& J.F.B. Pastore, Martianthus Harley \& J.F.B. Pastore, Hyptidendron Harley, Leptohyptis Harley \& J.F.B. Pastore, Condea Adans., Eplingiella Harley \& J.F.B. Pastore, Gymneia (Benth.) Harley\& J.F.B. Pastore, Cantinoa Harley\& J.F.B. Pastore e Mesosphaerum P.Browne.

As espécies pertencentes a esta família apresentam hábitos muito variados, correntemente arbustos, subarbustos, ervas, raro trepadeiras ou árvores, que podem ser perenes, bienais ou anuais. Suas folhas são opostas decussadas ou verticiladas, simples ou compostas, podendo ser inteiras, denteadas ou lobadas, pecioladas ou sésseis e sem estípulas (HARLEY, et al., 2004).

Comumentemente, as inflorescências são vistosas, axilares ou terminais, com ramos cimosos. As flores são andróginas, diclamídeas e zigomorfas. O cálice possui cinco sépalas unidas e persistentes, podendo ser tubuloso, campanulado ou infundiforme, com ápice bilabiado ou 5-10 denteado ou lobado. A corola tem cinco pétalas unidas, podendo ser tubulosa, campanulada ou infundibuliforme, com o ápice bilabiado. $O$ androceu possui quatro ou dois estames que estão inseridos no tubo da corola, os filetes são livres entre si, retos ou curvos e as anteras apresentam uma ou duas tecas. O gineceu é bicarpelar, sincárpico, com quatro lóculos, dois óvulos em cada carpelo, o ovário é súpero e apresenta um estilete do tipo ginobásico e dois estigmas. Nas Lamiaceae, o fruto é denominado núcula (BENTHAM, 1832-1836; HARLEY, et al., 2004). A polinização é efetuada por animais, precipuamente por vários insetos (HARLEY, et al., 2004).

As espécies da família Lamiaceae podem produzir muitas variedades de compostos secundários, contudo são conhecidas por apresentarem óleos essenciais presentes nos tricomas glandulares e nas superfícies das folhas e nas inflorescências (HARLEY, et al., 2004). Os óleos voláteis consistem singularmente de monoterpenoides, diterpenoides e sesquiterpenoides. Uma das classes de terpenoides mais encontradas nesta família são os diterpenóides, onde, vários destes compostos, apresentam características antibacteriana, antifúngica, além de possuir função ecológica nas plantas (HARLEY, et al., 2004; RICHARDSON, 1992). Os ácidos ursólico e oleanólico pertencente as classes dos triterpênicos, também se são encontrados nas Lamiaceae (BRUNETON, 2001). Vários estudos com espécies de Lamiaceae revelaram que alguns compostos químicos presentes 
nesta planta, podem estar envolvidos nas atividades antiedematogênica (COSTA, et al., 2008) e antifúngica (LEMES, et al., 2011), além de apresentarem atividades antiinflamatória, antioxidante, antitumoral (DALLA VECHIA, et al., 2009), antineoplásica (TOKUDA, et al., 1986) e hepatoprotetora (SARASWAT, et al., 1996). Alguns constituintes fenólicos também são encontrados nas Lamiaceae, como o estéres do ácido cafeico, a exemplo do ácido rosmarínico (HARLEY, et al., 2004; RICHARDSON, 1992), sendo que, o ácido rosmarínico demostrou atividades com ações farmacológicas, tais como, antibacteriana, anticomedogênica, anti-inflamatória, antioxidante, antiviral e neuroprotetora (PETERSEN, 1997).

A família Lamiaceae também se destaca por sua importância econômica e é abrangentemente utilizada para fins culinários, como por exemplo: Ocium Basilicum L. (alfavaca), Orinagum vulvare L. (orégano), Mentha x piperita L. (hortelã-pimenta), Mentha pulegium L. (poejo), Rosmarinus officinalis L. (alecrim), Salvia officinalis L. (sálvia) e Thymus vulgaris L. (tomilho); na perfumaria como Lavandula angustifólia Mill. (lavanda, alfazema); na preparação de bebidas, refrescos ou chás como Melissa officinalis L. (ervacidreira), Mentha spp. (hortelã) e Salvia hispânica L. (sálvia-azul) e Salvia splendens Sellow ex Roem. \& Schult. (sangue-de-adão) (HARLEY, et al., 2004), e na medicina popular, a M. piperita, é utilizada para alívio da congestão nasal e sinusite e externamente como analgésico em reumatismos (NUÑEZ; OBÓN DE CASTRO, 1992). Já a população nativa representando a América do Norte, utiliza-se a hortelã-pimenta para tratamento de desordens neurológicas, cardíacas, respiratórias e gastrointestinais (também utilizado pela população da América do Sul) (HARLEY; REYNOLDS, 1992). No estado de Minas GeraisBrasil, Leonurus sibiricus L. (macaé) é utilizada no tratamento da bronquite, M. officinalis como digestivo e calmante, Mentha arvensis L. (vick) contra congestão nasal, M. pulegium como calmante e contra a gripe e O. basilicum na constipação (OLIVAIRA, et al., 2010).

\subsection{Diabetes melitus}

Diabetes Melitus (DM) é o nome dado a um grupo de desordens com diferentes etiologias. Esta é caracterizada pelo distúrbio no metabolismo dos carboidratos, proteínas e lipídios causada pela completa ou relativa insuficiência da secreção e/ou ação da insulina. De acordo com a Organização Mundial da Saúde (OMS) a DM é geralmente classificada como Diabetes Melitus Insulino-Dependente (DMID) e Diabetes Melitus Não-InsulinoDependente (DMNID) (TABELA 1). 
O Diabetes Melitus Insulino-Dependente (DMID) ou tipo 1 caracteriza-se pela ausência da secreção de insulina por destruição total ou parcial das células $\beta$ do pâncreas. O tratamento consiste principalmente no uso diário de injeções de insulina e as possíveis complicações decorrentes da insulino terapia compreendem hipoglicemia, lipodistrofia, alergia local ou sistêmica e infecção nos locais de aplicação (CHAMPE, HARVEY, 1996; RANG et al, 2000; BRAGANÇA, 1996).

A insulina é um hormônio polipeptídico produzido pelas células $\beta$ das ilhotas de Langerhans no pâncreas. É composta por 51 aminoácidos arranjados em duas cadeias polipeptídicas, designadas $\mathrm{A}$ e $\mathrm{B}$, as quais estão ligadas por pontes dissulfeto (conectando os aminoácidos $A 7$ ao B7 e A20 ao B19). Uma terceira ponte dissulfeto na cadeia $A$ liga os resíduos $A 6$ e A11. Sua principal função é controlar o metabolismo intermediário, exercendo ações sobre o fígado, músculo e tecido adiposo. O efeito global da insulina consiste em conservar os combustíveis energéticos ao facilitar a captação, utilização e o armazenamento da glicose, dos lipídios e das proteínas (CHAMPE, HARVEY, 1996; RANG et al, 2000; BRAGANÇA, 1996).

TABELA 1: COMPARAÇÃO ENTRE OS DOIS TIPOS DE DIABETES

\begin{tabular}{|c|c|c|}
\hline & $\begin{array}{c}\text { DIABETES MELITUS } \\
\text { INSULINO-DEPENDENTE } \\
\text { (DMID) }\end{array}$ & $\begin{array}{l}\text { DIABETES MELITUS NÃO- } \\
\text { INSULINO-DEPENDENTE (DMNID) }\end{array}$ \\
\hline SINÔNIMO & Tipo 1 ou início juvenil & Tipo 2 ou início adulto \\
\hline IDADE DE INÍCIO & $\begin{array}{l}\text { Usualmente na infância ou } \\
\text { puberdade }\end{array}$ & Frequentemente acima de 40 anos \\
\hline TIPO FÍSICO & Magro & Obeso \\
\hline PREVALÊNCIA & $\begin{array}{l}10-20 \% \text { dos diabetes } \\
\text { diagnosticados }\end{array}$ & $80-90 \%$ dos diabetes diagnosticados \\
\hline $\begin{array}{c}\text { INSULINA } \\
\text { PLASMÁTICA }\end{array}$ & Baixa a ausente & Normal a elevada \\
\hline $\begin{array}{l}\text { DEFEITO OU } \\
\text { DEFICIÊNCIA }\end{array}$ & Células $\beta$ destruídas & $\begin{array}{c}\text { Incapacidade das células } \beta \text { em } \\
\text { produzir quantidades apropriadas de } \\
\text { insulina; } \\
\text { Resistência à insulina }\end{array}$ \\
\hline $\begin{array}{l}\text { COMPLICAÇÕES } \\
\text { AGUDAS }\end{array}$ & Cetoacidose & Coma hiperos molar \\
\hline CETOSE & Comum & Rara \\
\hline $\begin{array}{l}\text { TRATAMENTO } \\
\text { INSULINA }\end{array}$ & Sempre necessário & Usualmente não requerido \\
\hline $\begin{array}{l}\text { HIPOGLICEMIANTES } \\
\text { ORAIS }\end{array}$ & Não responsivo & Responsivo \\
\hline
\end{tabular}


Os efeitos metabólicos da insulina podem ser observados no metabolismo de carboidratos, lipídios e proteínas. No metabolismo dos carboidratos os efeitos da insulina são mais evidentes em três tecidos: fígado, músculo e tecido adiposo. No fígado, a insulina diminui a produção de glicose por inibir a gliconeogênese, diminui a degradação do glicogênio (glicogenólise) e estimula a síntese de glicogênio. No músculo, a insulina aumenta a síntese de glicogênio, aumenta a glicólise e aumenta a captação de glicose por aumentar o número de transportadores de glicose (Glut-4) na membrana plasmática. No tecido adiposo, a insulina aumenta a captação de glicose semelhante ao músculo. Assim, a administração intravenosa de insulina causa diminuição imediata na concentração da glicose no sangue. No metabolismo dos lipídios a insulina aumenta a síntese de ácidos graxos e a formação de triglicerídeos no tecido adiposo, enquanto inibe a lipólise, em parte através da desfosforilação (e, portanto, inativação) das lipases e, também provoca lipogênese no fígado. No metabolismo das proteínas a insulina estimula a entrada de aminoácidos nas células e aumenta a síntese de proteínas, na maioria dos tecidos (TABELA 2) (CHAMPE; HARVEY, 1996) (CHAMPE, HARVEY, 1996; RANG et al, 2000; BRAGANÇA, 1996).

TABELA 2: Resumo dos efeitos da insulina sobre o metabolismo de carboidratos, lipídios e proteínas no fígado, músculo e tecido adiposo.

\begin{tabular}{|c|c|c|c|}
\hline $\begin{array}{c}\text { TIPO DE } \\
\text { METABOLISMO }\end{array}$ & $\begin{array}{l}\text { CÉLULAS } \\
\text { HEPÁTICAS }\end{array}$ & TECIDO ADIPOSO & MÚSCULO \\
\hline $\begin{array}{l}\text { METABOLISMO DE } \\
\text { CARBOIDRATOS }\end{array}$ & $\begin{array}{l}\text { Diminuição da } \\
\text { glicogênese e } \\
\text { glicogenólise; } \\
\text { Aumento da } \\
\text { glicólise e } \\
\text { glicogênese }\end{array}$ & $\begin{array}{l}\text { Aumento da } \\
\text { captação de glicose } \\
\text { e síntese de glicerol }\end{array}$ & $\begin{array}{c}\text { Aumento da } \\
\text { captação de } \\
\text { glicose, da glicólise } \\
\text { e glicogênese }\end{array}$ \\
\hline $\begin{array}{l}\text { METABOLISMO DE } \\
\text { LIPÍDIOS }\end{array}$ & $\begin{array}{c}\text { Aumento da } \\
\text { lipogênese; } \\
\text { Diminuição da } \\
\text { lipólise }\end{array}$ & $\begin{array}{c}\text { Aumento da síntese } \\
\text { de triglicerídeos e } \\
\text { da síntese de } \\
\text { ácidos graxos }\end{array}$ & \\
\hline $\begin{array}{l}\text { METABOLISMO DE } \\
\text { PROTEÍNAS }\end{array}$ & $\begin{array}{l}\text { Diminuição da } \\
\text { degradação das } \\
\text { proteínas }\end{array}$ & & $\begin{array}{c}\text { Aumento da } \\
\text { captação de } \\
\text { aminoácidos e da } \\
\text { síntese de proteínas }\end{array}$ \\
\hline
\end{tabular}


No Diabetes Melitus Não-Insulino-Dependente (DMNID) ou tipo 2 existem no mínimo dois defeitos fundamentais: o primeiro é a diminuição na habilidade dos tecidos periféricos em responder a insulina (o que é denominado resistência à insulina) e o segundo é a deficiência funcional das células $\beta$ do pâncreas em secretar insulina, o que resulta numa hiperglicemia duradoura. O tratamento é baseado em medidas dietéticas, exercícios, agentes hipoglicemiantes orais e quando necessário doses moderadas de insulina. Os principais grupos de agentes hipoglicemiantes orais que reduzem a glicemia são assulfoniluréias, as biguanidas e os inibidores as $\alpha$-glicosidase. As sulfoniluréias exercem a principal ação sobre as células $\beta$ estimulando a secreção de insulina e reduzindo assim a concentração plasmática de glicose. Mas para exercerem esta função exigem a presença de células $\beta$ funcionais. É indicado para pacientes magros que possuem deficiência de insulina em maior grau. Seus efeitos colaterais são: hipoglicemia e ganho de peso corporal. Os principais fármacos encontrados no mercado são: tolbutamide, glibenclamida, clorpropamida e outros. O grupo das biguanidas não necessita das células $\beta$ funcionais. Sua ação é complexa e ainda não foi totalmente esclarecida. Sabe-se que elas aumentam a sensibilidade à insulina, reduzem a absorção intestinal de glicose, diminuem a gliconeogênese e aumentam a captação de glicose pelos tecidos periféricos. O principal efeito indesejável consiste em distúrbio gastrintestinal transitório. Exemplo: metformina. Os inibidores da a-glicosidase intestinal atuam no retardamento da absorção de carboidratos, reduzindo o aumento pós-prandial da glicemia. Os efeitos colaterais mais comuns são flatulência, fezes moles, diarréias, dores e distensões abdominais. Exemplo acarbose. Tanto as biguanidas e como os inibidores da a-glicosidase intestinal são indicados para pacientes com diabetes do tipo 2 que são obesos e não respondem ao tratamento apenas com a dieta (BRAGANÇA, 1996; CHAMPE; HARVEY, 1996; RANG, et al., 2000). Sendo assim, a busca e o desenvolvimento de compostos oralmente ativos que imitam ou aumentam as propriedades da insulina é uma tarefa urgente. Estes compostos orais poderiam substituir as dolorosas injeções de insulina no diabetes do tipo 1 e diminuir os efeitos colaterais dos agentes hipoglicemiantes orais no diabetes do tipo 2 .

\subsection{Aspectos hipoglicemiantes das lamiaceae}

Muitas espécies de plantas têm sido usadas etnofarmacologicamente ou experimentalmente para tratar dos sintomas do diabetes melitus (SAXENA, VIKRAM, 2004). Estas plantas representam mais de 725 gêneros em 183 famílias, estendendo-se 
fisiologicamente das algas marinhas e fungos para plantas, tais como as compostas. A distância filogenética entre este grupo de famílias é forte indicação da natureza variada de seus constituintes ativos (TABELA 3) (LAMBA ET AL., 2000). Entretanto, as análises posteriores revelaram grande variedade de mecanismos de ação que podem levar ao efeito hipoglicemiante, nem todos terapeuticamente úteis (HUO et al., 2003). Na região sudoeste do Marrocos, 37 plantas são usadas no tratamento do diabetes, porém devem ser utilizadas com muito cuidado, especialmente no diabetes tipo I, porque algumas dessas plantas medicinais podem provocar acidentes hipoglicêmicos (EDDOUKS, et al., 2002).

Os metabólitos isolados de Ocimum campechianum mil pertencente à família das Lamiaceae, rosmarinato e o ácido rosmarínico, causaram uma diminuição da glicose no sangue maior do que a causada pela acarbose, um resultado que parece estar relacionado com a sua forte atividade inibidora da a-glucosidase. Embora os flavonóides 5-demetil nobiletina e luteolina (isolados desta planta) não tenham demonstrado uma boa inibição da a-glicosidase, esses produtos diminuíram a glicemia no modelo in vivo, sugerindo um mecanismo anti-hiperglicêmico diferente (RUIZ-VARGAS, et al., 2019).

TABELA 3 - Famílias das plantas que possuem atividade hipoglicemiante

\begin{tabular}{ccc}
\hline Família & Espécies citadas & Total de espécies \\
\hline Fabaceae & 127 & 18.000 \\
Asteraceae & 98 & 21.300 \\
Lamiaceae & 36 & 3.500 \\
Limiaceae & 35 & 6.460 \\
Poaceae & 30 & 10.000 \\
Euphorbiaceae & 30 & 7.000 \\
\hline
\end{tabular}

Fonte: Marles e Farnsworth, 1995

O extrato de Salvia miltiorrhiza (SM) exibiu atividades antidiabéticas por meio do tratamento de doenças macro e microvasculares em experimentos pré-clínicos e ensaios clínicos através de uma melhoria da homeostase redox e inibição da apoptose e inflamação via regulação de Wnt / $\beta$-catenina, TSP-1 / TGF- $\beta 1$ / STAT3, JNK / PI3K / Akt, receptor B2 de cinina-Akt-GSK-3 $\beta$, AMPK $\beta$ / PGC-1 $\alpha$ / Sirt3, Akt / AMPK, TXNIP / NLRP3, TGF- $\beta 1$ / NF$\mathrm{KB}$, receptor de mineralocorticóide / $\mathrm{Na}+/ \mathrm{K}+$ - Caminhos de sinalização de ATPase, AGEs 
/ RAGE, Nrf2 / Keap1, CaMKKß / AMPK, AMPK / ACC, IRS-1 / PI3K e modulação dos canais $\mathrm{K}+-\mathrm{Ca} 2$ +, bem como influência de VEGF, NOS, AGEs, expressão de PPAR e agregação de hIAPP. Os efeitos antidiabéticos dessa erva podem estar relacionados a seus caracteres da Medicina Tradicional Chinesa de melhorar a circulação sanguínea e reviver a estagnação do sangue. Os principais ingredientes do SM incluíram os ácidos salvianólicos e tanshinonas diterpenoides, que foram bem estudados nos animais diabéticos. Estudos de toxicidade aguda e subaguda apoiaram a noção de que a SM é bem tolerada (JIA, et al., 2019).

\section{CONCLUSÃO}

A partir desses dados relatados acima, podemos concluir que diversas espécies da família Lamiaceae, possuem ação contra o Diabetes melitus e outras famílias com a filogenética próxima a esta família, também são usadas para o tratamento desta desordem.

\section{REFERÊNCIAS}

BENTHAM, G. Labiatum genera et species or a description of the genera and species of plants of the order Labiatae. ed. London: James Moyes: 1832-1836.

BRAGANÇA, L. A. R. Plantas Medicinais Antidiabéticas: uma abordagem multidisciplinar. 1. ed. Niterói: FLUMINENSE, E. D. U. F., 1996.

BRUNETON, J. Farmacogosia: fitoquímica e plantas medicinais. ed. Zaragoza: Acribia: 2001.

CHAMPE, P. C. , HARVEY, R. A. Bioquímica llustrada. 1. ed. Porto Alegre: MÉDICAS, A., 1996.

COSTA, H. N. R., SANTOS, M. C., ANCÂNTARA, A. F. C., SILVA, M. C., FRANÇA, R. C. , PILÓ-VELOSO, D. Constituintes químicos e atividades antiedematogênica de Petodon radicans (Lamiaceae). Química Nova. v. 31, n. p. 744-750, 2008.

DALLA VECHIA, L., GNOATTO, S. C. , GOSMANN, G. Derivados oleananos e ursanos e sua importância na descoberta de novos fármacos com atividade antitumoral, antiinflamatória e antioxidante. Química Nova. v. 32, n. p. 1245-1252, 2009. 
EDDOUKS, M., MAGHRANI, M., LEMHADRI, A., OUAHIDI, M. L. , JOUAD, H. Ethnopharmacological survey of medicinal plants used for the treatment of diabetes mellitus, hypertension and cardiac diseases in the south-east region of Morocco (Tafilalet). Journal of ethnopharmacology. v. 82, n. 2-3, p. 97-103, 2002.

Flora do Brasil 2020 em construção. Jardim Botânico do Rio de Janeiro. Disponível em: <http://floradobrasil.jbrj.gov.br/>. Acesso em: 16 Março 2018.

HARLEY, R. M., FRANÇA, F. Plantas raras no Brasil. ed. Conservação Internacional/Universidade Estadual de Feira de Santana, Belo Horizonte: 2009.

HARLEY, R. M., PASTORE, J. F. B. A genetic revision and new combinations in the Hyptidinae (Lamiaceae), based on molecular and morphological evidence. Phytotaxa. v. 58, n. 1, p. 2012.

HARLEY, R. M., REYNOLDS, T. Advances in Labiatae Science. ed. 1992.

HARLEY, R. M. An Explosive Mechanism in Eriope crassipes, a Brazilian Labiatae. Biological Journal of the Linnean Society 3. v. n. p. 159-164, 1971.

HARLEY, R. M., ATKINS, S., BUDANTSEV, A. L., CANTINO, P. D., CONN, B. J., GRAYER, R., HARLEY, M. M., KOK, R., KRESTOVSKAJA, T., MORALES, R., PATON, A. J., RYDING, O., UPSON, T., LABIATAE. IN: KADEREIT, J. W. , KUBITZKI, K. E. The families and genera of vascular plants. ed. Berlin: Springer: 2004.

HARLEY, R. M., FRANÇA, F., SANTOS, E. P. , SANTOS, J. S. in: Catálogo de Plantas e Fungos do Brasil. ed. ANDREA JAKOBSON ESTÚDIO, I. D. P. J. B. D. R. D. J., RIO DE JANEIRO, 2010.

HEYWOOD, V. H. Flowering Plants of the World. ed. Oxford: Oxford university press: 335, 1979.

JIA, Q., ZHU, R., TIAN, Y., CHEN, B., LI, R., LI, L., WANG, L., CHE, Y., ZHAO, D., MO, F., GAO, S. , ZHANG, D. Salvia miltiorrhiza in diabetes: A review of its pharmacology, 
phytochemistry, and safety. Phytomedicine: international journal of phytotherapy and phytopharmacology. v. 58, n. p. 152871, 2019.

JUDD, W. S., CAMPBELL, C. S., KELlOG, E. A., STEVENS, P. F., DONOGHUE, M. G. Sistemática Vegetal: em enfoque filogenético. 3. ed. Porto Alegre, Artmed: 492-494, 2009.

LEMES, G. F., FERRI, P. H., LOPES, M. N. Constituíntes químicos de Hyptidendron canum (Pohl ex Benth.) R. Harley (Lamiaceae). Química Nova. v. 24, n. p. 39-42, 2011.

NUÑEZ, D. R., OBÓN DE CASTRO, C. Advances in Labiatae Science. Royal Botanic Gardens. v. n. p. 445-473, 1992.

OLIVAIRA, H. B., KFFURI, C. W., CASALI, V. W. D. Ethnopharmacological study of medicinal plants used in Rosário da Limeira, Minas Gerais, Brasil. Revista Brasileira de Farmacognosia. v. 20, n. p. 256-260, 2010.

PATON, A., RYDING, O. Hanceola, Siphocranion and Isodon and their position in the Ocimeae (Labiatae). Kew Bull. v. 53, n. 3, p. 723-731, 1998.

PETERSEN, M. Cytochrome P450-dependent hydroxylation in the biosynthesis of rosmaric acid in Coleus. Phytochemistry. v. 45, n. p. 1165-1172, 1997.

RANG, H. P., DALE, M. M., RITTER, J. M. Farmacologia. 1. ed. Rio de Janeiro: KOOGAN, G., 2000.

RICHARDSON, P. M. Advances in Labiatae Science. ed. Royal Botanic Gardens: 291-297, 1992.

RUIZ-VARGAS, J. A., MORALES-FERRA, D. L., RAMIREZ-AVILA, G., ZAMILPA, A., NEGRETE-LEON, E., ACEVEDO-FERNANDEZ, J. J., PENA-RODRIGUEZ, L. M. alphaGlucosidase inhibitory activity and in vivo antihyperglycemic effect of secondary metabolites from the leaf infusion of Ocimum campechianum mill. Journal of ethnopharmacology. v. 243, n. p. 112081, 2019. 
SARASWAT, B., VISEN, P. K. S., DAYAL, R., AGARWALL, D. P. , PATNAIK, G. K. Protective action of ursolic acid against chemical induced hepato-toxicity in rats. Indian Journal Pharmacology. v. 28, n. p. 232-239, 1996.

SUDDE, S. A. A taxonomic revision of tribe Ocimeae Dumort (Labiatae) in continental South East Asia. Disponível em: Acesso em: 30 maio 2018.

TOKUDA, H., OHIGASHI, H., KOSHIMIZU, K., ITO, Y. Inhibitory effects of ursolic and oleanolic acid on skin tumor promotion by 12-O-tetradecanoylphorbol-13-acetate. Cancer letters. v. 33, n. 3, p. 279-285, 1986.

Word Health Organization. 23. ed. Manila: PUBLICATIONS, W. R., 1998.

Word Health Organization. ed. Manila: 1993.

YUNES, R. A., CALIXTO, J. B. Plantas medicinais sob a ótica da química medicinal moderna. ed. Chapecó: ARGOS, 2001. 